\title{
Warum Wissenschaft wichtig ist - ein Forschungswettbewerb als Vermittlungsformat
}

\author{
Friederike Schneider
}

Die Körber-Stiftung schreibt jedes Jahr unter der Schirmherrschaft des Bundestagspräsidenten den Deutschen Studienpreis aus, einen Forschungswettbewerb, bei dem Promovierte für fachlich herausragende Dissertationen ausgezeichnet werden, die zugleich für die Gesellschaft besonders relevant sind. Mit den ihm zugrunde liegenden Prinzipien und in seiner gesamten Konzeption ist der Wettbewerb dazu geeignet, der aktuellen Vertrauenskrise zwischen Wissenschaft und Gesellschaft entgegenzuwirken.

Denn mit diesem Preis zeichnen wir gute, gesellschaftlich relevante Wissenschaft aus und rücken sie ins Licht der Öffentlichkeit. Hinter der Initiative steht die Überzeugung von der alten aufklärerischen Idee, nach der eine Gesellschaft, die wissenschaftliches Wissen zur Verfügung hat und die damit möglichst gut und substanziell informiert ist, die besseren Entscheidungen trifft. Anders gesagt: Gelebte Demokratie ist ohne Wissenschaftlichkeit nicht möglich. Der Deutsche Studienpreis ist nicht nur ein Bekenntnis zu dieser Bedeutung der Wissenschaft für die Gesellschaft. Er zeigt auch, dass es an den deutschen Hochschulen und Forschungsinstituten eine junge Generation von Wissenschaftlerinnen und Wissenschaftlern gibt, die sich diese Rolle bewusst zu eigen macht.

Konkret geht es uns darum, junge Forscherinnen und Forscher zu fördern, die fachlich herausragend sind und die zugleich als Wissenschaftler gesellschaftliche Verantwortung übernehmen. Und es geht uns darum, junge

F. Schneider $(\bowtie)$
Körber-Stiftung, Hamburg, Deutschland
E-Mail: schneider@koerber-stiftung.de 
Wissenschaftlerinnen und Wissenschaftler zu fördern, die bereit und in der Lage sind, die Inhalte ihrer Forschungen über die Grenzen ihrer eigenen Fachcommunity hinaus zu vermitteln und in die gesellschaftliche Debatte einzubringen. Damit soll der Preis in die Wissenschaft hineinwirken und diese Haltung im Wissenschaftssystem verstärken, indem er sie beim Nachwuchs fördert.

Zugleich soll er eine Plattform für die Leistung junger Wissenschaftlerinnen und Wissenschaftler sein und ihnen und ihren Forschungsbefunden öffentliche Aufmerksamkeit verschaffen. Ziel ist dabei, anhand der preisgekrönten Einzelbeispiele der Öffentlichkeit zu zeigen, dass Wissenschaftlerinnen und Wissenschaftler sich mit einer Vielzahl von Themen befassen, an deren Bearbeitung die Gesellschaft ein Interesse hat. Der Deutsche Studienpreis macht also deutlich, wozu Wissenschaft ganz praktisch von Nutzen ist, warum sie für die Gesellschaft wichtig ist.

Dies leuchtet am konkreten Beispiel ganz unmittelbar ein - etwa anhand der Forschungsarbeit unserer Preisträgerin Elisabeth Wilhelm, die als Ingenieurin ein Computerdisplay für Blinde entwickelt hat, das Bilder und grafische Darstellungen mit den Fingern fühlbar macht. Oder am Beispiel der Forschungsarbeit der Politikwissenschaftlerin Julia Strasheim, die Gelingensbedingungen von Übergangsregierungen nach militärischen Konflikten erarbeitet hat. Oder anhand der Analyse der Literaturwissenschaftlerin Silke Braselmann, die darlegen konnte, wie fiktionale Darstellungen bei der Verarbeitung von Schulamokläufen helfen.

Das öffentliche Interesse an den Forschungsbefunden junger Wissenschaftlerinnen und Wissenschaftler muss man allerdings realistisch einschätzen. Ein Promotionspreis wird zunächst einmal als eine Angelegenheit von eher innerwissenschaftlicher Bedeutung wahrgenommen, bei der es darum geht, den Nachwuchs zu ermutigen und zu fördern. Spektakuläre Durchbrüche werden an anderer Stelle erwartet. Die traut man vor allem der Liga der Max-Planck-Direktoreninnen und -Direktoren, Forschungsgruppenleitenden und Exzellenzcluster-Sprecherinnen und -Sprechern zu - was in der Größenordnung ja auch richtig ist, denn selbstverständlich ist eine Dissertation ein begrenztes Forschungsvorhaben. Dementsprechend halten sich die Erwartungen bei Forschungsarbeiten, die mit einem Promotionspreis ausgezeichnet werden, in Grenzen und das öffentliche Interesse damit zunächst einmal auch.

Die Vergabe des Preises, der Versand einer Pressemitteilung sowie begleitende Tweets und Posts allein reichen also nicht aus, wenn man mit den Forschungsergebnissen tatsächlich wahrnehmbar zur öffentlichen Debatte beitragen möchte. Wir haben daher für den Deutschen Studienpreis 
über die Jahre unterschiedliche Formate entwickelt. Einige davon sollen im Folgenden dargestellt werden, wobei unsere Online-Formate hier entfallen, da dieses Thema im vorliegenden Buch an anderer Stelle ausführlicher erläutert wird.

\section{Deutscher Studienpreis der Körber-Stiftung}

Die Körber-Stiftung schreibt den Deutschen Studienpreis jedes Jahr bundesweit unter der Schirmherrschaft des Bundestagspräsidenten aus. Teilnahmeberechtigt an diesem Wettbewerb sind junge Wissenschaftlerinnen und Wissenschaftler aller Fachdisziplinen und aller Hochschulen, die ihre Dissertation mit dem Prädikat summa oder magna cum laude abgeschlossen haben. Jedes Jahr nehmen rund 400 Promovierte teil. Die Jurierung und die Preisvergabe erfolgen in drei Fachsektionen: den Natur- und Technikwissenschaften, den Sozialwissenschaften und den Kultur- und Geisteswissenschaften. In jeder Sektion wählt die Jury einen ersten und zwei zweite Preisträger aus insgesamt werden also neun Preise vergeben. Entscheidendes Kriterium ist die gesellschaftliche Relevanz.

In unserem Bemühen, die Preisträgerarbeiten öffentlich sichtbar zu machen, ist klassische Medienarbeit nach wie vor ein wichtiger Baustein. Zwar sind die klassischen Medien nicht mehr Gatekeeper zur breiten Öffentlichkeit, aber sie bieten nach wie vor einen wichtigen Zugang zu einem großen Teilbereich der Öffentlichkeit und sie haben vor allem das Potenzial entscheidend zur Reputation beizutragen - von einzelnen Wissenschaftlern und deren Instituten, aber auch von Themen. Ein eigener Meinungsbeitrag auf ZEIT Online, ein Bericht in der Frankfurter Allgemeinen Zeitung oder ein Interview im Deutschlandfunk zeigen, dass die jeweilige Redaktion gerade diese Wissenschaftlerin oder diesen Wissenschaftler als maßgeblichen Experten wahrgenommen hat oder eben dieses Thema wichtig genug fand, um ihm einen angemessenen Platz in der Zeitung bzw. kostbare Sendezeit im Programm einzuräumen. Als entsprechende Anerkennung erleben unsere Preisträgerinnen und Preisträger diese Medienanfragen und deren Institute und Hochschulen begleiten die Auftritte mit Wohlwollen.

Es wird gelegentlich kritisiert, in der Wissenschaft herrsche die Auffassung vor, Sichtbarkeit außerhalb der Zitationsindizes sei die falsche Währung und Medienpräsenz ein Zeichen, dass man sich vom Eigentlichen hat ablenken lassen. Diese Erfahrung machen wir mit dem Deutschen Studienpreis weder bei den jungen Wissenschaftlerinnen und Wissenschaftlern selbst noch bei deren Vorgesetzten in den Instituten. Zeitbudgets im Rahmen der Arbeitszeit gibt es für die Bearbeitung von Medienanfragen 
allerdings zumeist keine. Das heißt, die jungen Wissenschaftlerinnen und Wissenschaftler müssen in aller Regel selbst schauen, wie sie diese Aktivitäten mit ihrer wissenschaftlichen Arbeit vereinbaren. Der Austausch mit der Gesellschaft gerät daher letztlich eher zur Privatsache. Ein Interview wird gegebenenfalls noch tagsüber im Institut gegeben, die Vorbereitung erfolgt aber abends zu Hause - und das Verfassen eines eigenen Medienbeitrags sowieso. Kommunikation kostet Zeit, die - wenn der Dialog zwischen Wissenschaft und Gesellschaft gelingen soll - künftig stärker in den Wissenschaftsbetriebs einbezogen werden sollte, was sich letztlich auch in den innerwissenschaftlichen Anerkennungsmechanismen widerspiegeln müsste.

Obwohl dies aktuell kaum der Fall ist, gibt es bei den jungen Wissenschaftlerinnen und Wissenschaftlern durchaus Interesse, das eigene Forschungsthema über die klassischen Medien in die Öffentlichkeit zu tragen.

Diese interessieren sich zwar nicht per se für Promotionspreise, aber sie interessieren sich für spannende, aktuelle Forschungsthemen, die zudem einen Preis bekommen haben. So herum funktioniert die Logik bei der Vermittlung von Nachwuchsforschung. Deswegen läuft die Pressearbeit wie die übrige Öffentlichkeitsarbeit für den Deutschen Studienpreis fast ausschließlich über die Forschungsthemen. Wir sprechen gezielt passende Redaktionen zu den jeweils ausgezeichneten Beiträgen an, um ihnen unsere Preisträgerinnen und Preisträger für eine Berichterstattung, ein Interview oder als Autoren nahezulegen.

Am vielversprechendsten ist dies, wenn der Preisträger oder die Preisträgerin zu einem Thema geforscht hat, das ohnehin auf der Agenda steht und für das die Redaktionen auf der Suche nach Experten sind. Wissenschaft kommt ja weniger aus Anlass aktueller Forschungsergebnisse in die Medien, sondern viel häufiger deshalb, weil ein Ereignis auf der öffentlichen Agenda steht, zu dem wissenschaftliches Expertenwissen gefragt ist - etwa eine Grippewelle, ein extremes Hochwasser oder eine politische Krise.

Den in dieser Hinsicht spektakulärsten Fall gab es beim Deutschen Studienpreis im Zusammenhang der Umbrüche in Ägypten vor einigen Jahren: Die Zeithistorikerin und Politikwissenschaftlerin Annette Ranko wurde damals für ihre Forschungsarbeit zum Weltbild der ägyptischen Muslimbrüder ausgezeichnet. In der Nacht nach der Sitzung, in der die Studienpreis-Jury über die Preisränge entschieden hatte, wurde der ägyptische Präsident Mursi, ein Muslimbruder, vom Militär in Kairo nach Großdemonstrationen abgesetzt. Damit stand Ägypten und die Organisation der Muslimbrüder über Nacht in allen Redaktionen auf der Agenda. Wir haben 
damals sofort mit der Kommunikationsarbeit begonnen und Anette Ranko war über Wochen in allen großen Medien als Expertin präsent.

Dass das Thema so eindeutig auf der Tagesordnung steht, die Expertise der Wissenschaftlerin so genau passt und es - denn auch das ist bei einem Nachwuchswissenschaftler wichtig - nicht viele andere in den Redaktionen bereits bekannte Experten zum Thema gibt, ist natürlich der Ausnahmefall. Wir haben neun Preisträgerinnen und Preisträger pro Jahr, also insgesamt neun Themen, die selbstverständlich bei Weitem nicht alle direkt anschlussfähig an die tagesaktuellen Top-Ereignisse sind. Wir betreiben hier über einen gewissen Zeitraum ein Monitoring. Das heißt, wir beobachten, welche Themen in der öffentlichen Debatte sind, und prüfen, inwiefern die Arbeiten unserer Preisträger hierzu passen bzw. einen neuen Aspekt hinzuliefern könnten.

Ein Beispiel ist hier die Forschungsarbeit der Juristin Anna Beckers. Sie hatte sich in ihrer Dissertation mit freiwilligen Selbstverpflichtungen globaler Unternehmen befasst. Ihr Beispielfall war der Einsturz einer Textilfabrik im Jahr 2013 in Bangladesch. Der westliche Großkonzern, der dort produzieren ließ, hatte sich auf die Einhaltung von Arbeitsschutzstandards verpflichtet und mit dieser Selbstverpflichtung auch für den Verkauf seiner Produkte geworben. Rechtlich blieb es folgenlos, dass er dieser Selbstverpflichtung so offensichtlich nicht nachgekommen war. Wie eine rechtliche Haftung hier künftig in Absatzländern solcher Unternehmen erreichbar wäre, war Gegenstand der Arbeit unserer Preisträgerin.

Ein relevantes Thema - allerdings zunächst darum nicht sofort attraktiv für die Medien, weil der Beispielfall aus der Dissertation bereits einige Jahre zurücklag. Ein anderer Weltkonzern, der sich auf Standards, in diesem Fall Umweltstandards, verpflichtet und diese nicht eingehalten hatte, war allerdings zum Zeitpunkt der Auszeichnung unserer Preisträgerin sehr präsent in den Medien: der VW-Konzern. Die rechtlichen Erörterungen von Frau Beckers trafen auch hier zu und wir konnten sie als Expertin und Kommentatorin für dieses Thema sehr erfolgreich an die Medien vermitteln. Auf diesem Wege konnte die Juristin einen neuen wichtigen Aspekt in die öffentliche Debatte bringen und ihre Vorschläge für Rechtsreformen in der Haftung bei Selbstverpflichtungen breit öffentlich zur Diskussion stellen.

Formate, in denen unsere Preisträger selbst zu Wort kommen - wie Interviews und selbst verfasste Artikel -, sind übrigens die häufigste Form, in der sie in den Medien zu finden sind. Es sind oft die Meinungs- und Kommentarseiten und die Rubriken für externe Autoren, in denen unsere Preisträger erscheinen. Im Hörfunk und Fernsehen sind es Live-Interviews 
und Talkformate. Die Rolle der Kommunikationsabteilung - die in diesem Fall die Körber-Stiftung für die jungen Wissenschaftlerinnen und Wissenschaftler einnimmt - ist eine vermittelnde. Ein echter Dialog zwischen Wissenschaft und Gesellschaft kann sich aber nur entwickeln, wenn Wissenschaftlerinnen und Wissenschaftler selbst kommunizieren und auch für das weitere Gespräch offen sind. Insofern tragen Imagebroschüre zum Austausch von Wissenschaft und Gesellschaft relativ wenig bei.

Wir versuchen unseren Preisträgerinnen und Preisträgern auch die Möglichkeit zu geben, mit politischen Entscheidern über ihre Forschungsergebnisse ins Gespräch zu kommen. Zum Teil geschieht dies über die außenpolitischen und die gesellschaftspolitischen Aktivitäten, Netzwerke und Gesprächsformate der Körber-Stiftung. Wir prüfen also systematisch, für welche anderen unserer Stiftungsaktivitäten die wissenschaftliche Expertise unserer Preisträgerinnen und Preisträger interessant sein könnte.

Gemeinsam mit dem Forschungsausschuss des Deutschen Bundestages haben wir zudem ein eigenes Format entwickelt und laden dazu jeweils gemeinsam mit dem Ausschussvorsitzenden ein: den „Salon junge Wissenschaft“, der seit Neuestem unter dem Titel „Eine Stunde junge Wissenschaft" neu aufgenommen wurde. Die Grundidee ist ein schlankes einstündiges Event, bei dem jeweils ein Preisträger oder eine Preisträgerin Parlamentariern des Deutschen Bundestages die eigene Forschungsarbeit vorstellt und diese mit ihnen diskutiert.

Die Zahl der teilnehmenden Abgeordneten an den Veranstaltungen ist sehr unterschiedlich - meistens kommen Abgeordnete, die sich in ihrer parlamentarischen Arbeit fachlich mit dem jeweils präsentierten Thema befasst haben, aber auch andere Interessierte. Für die jungen Wissenschaftlerinnen und Wissenschaftler ist dies nicht nur eine Chance, ihre Forschungsergebnisse an entscheidender Stelle zu Gehör zu bringen, sondern ebenso spannend, dann wiederum mit den Realitäten der politischen Praxis in Kontakt zu kommen.

Ein gutes Beispiel für einen Salon mit dieser Wirkung war die Veranstaltung, in der der Mediziner und Medizinethiker Ralf Jox auftrat. Er hatte im Rahmen seiner Dissertation eine Erhebung zur passiven Sterbehilfe auf deutschen Intensivstationen durchgeführt. Diese ergab, dass Ärzte, wenn sie therapeutische Maßnahmen bei Sterbenden schließlich einstellen, dies in dem Gefühl tun, in einer ethischen und rechtlichen Grauzone zu agieren. Zu dem Zeitpunkt, als der „Salon junge Wissenschaft“ mit Ralf Jox stattfand, lag eine Gesetzesänderung zur passiven Sterbehilfe noch nicht lange 
zurück. Diese sah unser Preisträger als unzureichend an und vertrat das auch im Salon. Das war ein sehr intensiver Nachmittag und ein ausgesprochen ernsthafter Austausch, der für beide Seiten instruktiv war. Ralf Jox hat sich weiter mit dieser Thematik befasst und einige Jahre später gemeinsam mit drei weiteren Kollegen einen Gesetzesvorschlag zur passiven Sterbehilfe vorgelegt, den eine fraktionsübergreifende Gruppe um Karl Lauterbach und Peter Hintze zur Grundlage für einen Reformvorschlag machte, über den Ende 2015 im Bundestag entschieden wurde.

Über die politischen Entscheider hinaus geht es uns aber auch darum, ein breites Publikum mit Veranstaltungen anzusprechen und ihm die Forschungsbefunde unserer Preisträgerinnen und Preisträger zu vermitteln. Ein Format hierfür haben wir sieben Jahre lang gemeinsam mit dem Deutschlandfunk veranstaltet. Wir haben in Kooperation mit der Redaktion Podien organisiert, die jedes Mal ein Preisträgerthema zum Ausgangspunkt einer Debatte nahmen. Unser Preisträger oder unsere Preisträgerin war dabei jeweils einer der Podiumsgäste und diskutierte seine/ihre Forschungsbefunde mit Experten aus Politik und Praxis. Die Veranstaltungen fanden in unserem Veranstaltungszentrum, dem KörberForum, vor Publikum statt und wurden live im Deutschlandfunk im Rahmen der Reihe „Zur Diskussion“ gesendet. Hier diskutierte z. B. zuletzt unser Preisträger, der Jurist Tom Pleiner, mit dem Grünen-Politiker Robert Habeck und dem Geschäftsführer des Netzbetreibers Tennet über Energiewende und Netzausbau. Im Anschluss an die Live-Sendung folgte eine Debatte mit dem Saalpublikum - zumeist rund 300 Personen. Im Live-Auftritt kamen die Nachwuchswissenschaftler, so zeigt das Feedback von Publikum und Hörern, besonders gut an: Dass die Dissertation ein begrenztes Forschungsprojekt ist, was von den jungen Wissenschaftlerinnen und Wissenschaftlern auch entsprechend kommuniziert wurde, erwies sich nicht als Nachteil. Im Gegenteil: Gerade der ehrliche Umgang mit den Grenzen der Reichweite der eigenen Forschung kam in den Saaldebatten beim Publikum als Ernsthaftigkeit an und trug eher zu einer intensiven Diskussion bei.

Unser Fazit nach über zehn Jahren Deutscher Studienpreis lautet: Das Vertrauen der Gesellschaft in die Wissenschaft fördert man am besten, wenn man guter gesellschaftlich relevanter Wissenschaft eine Plattform gibt nicht in Form von Top-down-Vermittlungsformaten, sondern als ernsthaftes Gesprächsangebot. Im Idealfall entsteht dabei der nach unserer Auffassung zutreffende Eindruck, dass Wissenschaft eine wesentliche, wenn nicht die Instanz zur Lösung anstehende Zukunftsfragen ist. 
Friederike Schneider studierte an der Universität Hamburg Ostslawistik und Politikwissenschaft. Im Anschluss an das Studium absolvierte sie ein Volontariat in einer Kommunikationsagentur und begann danach ihre Tätigkeit bei der Körber-Stiftung - zunächst als wissenschaftliche Mitarbeiterin und später als Programm-Managerin für den Deutschen Studienpreis. Seit 2017 ist sie Programmleiterin für das Feld Hochschule im Bereich Wissenschaft der Körber-Stiftung.

Open Access Dieses Kapitel wird unter der Creative Commons Namensnennung 4.0 International Lizenz (http://creativecommons.org/licenses/by/4.0/deed.de) veröffentlicht, welche die Nutzung, Vervielfältigung, Bearbeitung, Verbreitung und Wiedergabe in jeglichem Medium und Format erlaubt, sofern Sie den/die ursprünglichen Autor(en) und die Quelle ordnungsgemäß nennen, einen Link zur Creative Commons Lizenz beifügen und angeben, ob Änderungen vorgenommen wurden.

Die in diesem Kapitel enthaltenen Bilder und sonstiges Drittmaterial unterliegen ebenfalls der genannten Creative Commons Lizenz, sofern sich aus der Abbildungslegende nichts anderes ergibt. Sofern das betreffende Material nicht unter der genannten Creative Commons Lizenz steht und die betreffende Handlung nicht nach gesetzlichen Vorschriften erlaubt ist, ist für die oben aufgeführten Weiterverwendungen des Materials die Einwilligung des jeweiligen Rechteinhabers einzuholen. 